\title{
GUÍA BÁSICA PARA ESCRIBIR UN ARTículo PARA PUBLICACIONES CIENTÍFICAS
}

\author{
'Sonia Constanza Concha S., Martha Julianu Rodiziguez G. \\ 'Odontóloga. U. Santo Tbmás, Lspecialista en Fducación y Comunicación para la Salud y del Bienestar, U. Industrial de Santander, \\ Aspirante a Magister en Epideniologio. U. Industrial de Sancander. Docente, U. Santo Tomás y U. Industrinl de Santandes: \\ ¿Odontäloga, U. Javeriana, Especialista en Odantopediatría. C.E.S. Docente. U. Santo Tomás
}

Autor responsable de correspondencia: Sonia Constanza Concha $S$.

Correo ulectrónicu: sncocosa@yahoo.com

\begin{abstract}
RESUMEN
Divulgar la información cientifica es una actividad clave en un proceso investigativo; el articulo cientifico. el articulo de revisión y los reportes de caso son una altemativa para hacerlo. Fl objetivo del presente trabajo fue brindar una guía básica para escribir mejor y aspirar a publicar lo que se escribe. [Concha SC. Rodríguez M]. Guia básica para escribir un articulo para publicaciones cientificas. Ustasalud Odontologia 2005; 4: $48-55]$
\end{abstract}

Palabras claves: Articulo científico, Articulo de revision, Reporte de caso.

\section{A BASIC GUIDE TO WRITE A PAPER FOR SCIENTIFIC JOURNAI.}

\begin{abstract}
To disclose the scientific information is a key activity in an investigative process; the scientific article, the review paper and the case reports are the alternatives. The objective of the present work was to offer a basic guide to write a puper and look for its publication.
\end{abstract}

Key words: Original paper, Review aricle, Case report.

Recibldo para publicación: 15 de mayo de 2005. Aceptado para publicación: 5 de junıo de 2005 .

\section{INTRODUCCIÓN}

La investigación científica es una actividad inherente al ejercicio odontológico, permite trascender de simples técnicos a verdaderos profesionales. Uno de los aspectos básicos de la investigación científica es la divulgación del conocimiento ya que un experimento científico, por espectacular que sean sus resultados, no termina hasta que éstos se publiquen. El investigador está obligado a presentar un informe escrito de lo que hizo, por qué lo hizo, como lo hizo y lo que aprendió al hacerlo. ${ }^{1,2}$

A nivel técnico investigativo es importante destacar dos formas fundamentales para realizar la divulgación de los resultados y estos son la presentación oral de una investigación y el artículo científico. Para cada una de estas formas de divulgación, es importante resaltar la necesidad de conocer aspectos básicos que permitan una mejor presentación y claridad del conocimiento que se desea dar a conocer. ${ }^{2,3}$
El artículo es un informe escrito y publicado que describe los resultados derivados del proceso investigativo; de acuerdo con la información que presenta se puede catalogar como artículo original, de revisión o reporte de caso. En el original, la información es el producto de la recolección de los datos por parte del investigador y la presentación sistemática de sus resultados. Este debe informar sobre un avance específico, mostrar evidencia, ser consistente desde el punto de vista de la lógica, ser reproducible, dar referencias de investigaciones previas y estar disponible. ${ }^{*}$

Los articulos que resumen, analizan, evalúan, sintetizan la información ya publicada se denominan artículos de revisión." Estos pueden ser de dos tipos de acuerdo con su nivel de análisis:

- Revisión simple: se realiza una descripción de las referencias.

- Revisión sistemática: se valora la evidencia científica y se aplican los parámetros de la odontologia basada en la evidencia. ${ }^{5}$ 
En algunas publicaciones científicas, tienen en cuenta los artículos basados en el reporte de casos en los que se manifiestan hallazgos clinicos novedosos en una persona o en un grupo de personas con una condición particular. ${ }^{6}$

Tanto los articulos originales, los de revisión y los reportes de caso cumplen una función especifica y tienen elementos particulares que deben incluirse en el manuscrito final para lograr el sustento científico. ${ }^{2}$

Sin embargo, las guías básicas para la elaboración de este tipo de articulos no se conocen muy bien y esto es particularmente evidente en los nuevos investigadores y profesionales; de ahi la importancia de brindar elementos básicos que permitan elaborar articulos que cumplan con los requisitos técnicos establecidos para su publicación.

En este sentido, el objetivo de la presente revisión es brindar una guia básica para escribir artículos originales, de revisión y reportes de caso. Es necesario aclarar que los rasgos particulares exigidos, podrian cambiar de una publicación a otra y que es indispensable tener en cuenta el aparte sobre las indicaciones para los autores de la revista en la que se desea publicar; de esta manera, se incrementa la posibilidad de publicación.'

\section{RAZONES PARA ESCRIBIR}

Además de avanzar en el conocimiento, escribir y publicar los resultados de una investigación implica un avance para la institución en la que ésta se desarrolló y una satisfacción propia. ${ }^{7}$

Las publicaciones de alta calidad aumentan el prestigio de un departamento o de una institución. En algunas ocasiones se incrementa el apoyo financiero destinado a investigación ya sea por parte de la institución o de organismos gubernamentales.?

Quizás, la razón más importante para escribir es la satisfacción propia. Se adquieren varias habilidades al adoptar esta práctica como una disciplina:

- Aumentan las oportunidades de promoción y desempeño. Actualmente, la mayoría de los currículos exigen publicaciones.

- Mejora el perfil profesional.

- Aumentan las habilidades en investigación.

- Obtiene reconocimiento nacional y/o internacional.

\section{ASPECTOS GENERALES A CONSIDERAR AL ES CRIBIR UN ARTÍCULO}

La escritura debe ser directa, precisa, impersonal y en pretérito. Inicialmente, es útil escribir por etapas. En una de éstas se realiza un borrador sin preocuparse por detalles que serán mejorados en una etapa posterior. ${ }^{1.59}$

Cada autor puede desarrollar su propio estilo, pero en todo caso deberá ser claro y fácil de leer. Es necesario evitar la jerga y los modismos propios de cada región y la repetición de la información. ${ }^{2}$

La ortografia y la gramática son aspectos fundamentales, no hay excusa válida que sustente esta falla, particularmente ahora que existen mecanismos de corrección en la mayoria de los procesadores de texto. ${ }^{3}$

En algunas revistas es aceptado el uso de abreviaturas pero en otras no; en algunas sólo las abreviaturas interna-cionalmente estandarizadas. Las unidades de medidas se pueden expresar como abreviaturas, de igual forma tambien se aceptan en las tablas donde los espacios son limitados, en este caso, la abreviatura deberá ser explicada en el pie de la tabla. ${ }^{2}$ Si se usan abreviaturas en el texto, siempre se debe escribir la palabra completa al mencionarla por primera vez y. al lado de esta, y entre paréntesis la abreviatura correspondiente, de ahí en adelante puede mencionarse sólo la abreviatura sin paréntesis.

Es útil leer el manuscrito en voz alta. Una armoniosa combinación de vocablos le permite descubrir defectos de tono y estilo que pueden pasar inadvertidos en una simple lectura visual."

En relación con la escritura de los números, se debe tener en cuenta que los dígitos $(0$ - 9) no se escriben con números sino con letras; a partir del 10, se escriben con números. Si una frase inicia con un número, es necesario escribirlo en letras.

la presentación final del manuscrito se realiza a una columna y las tablas y gráficos se ubican al final del escrito. El número asignado a cada tabla o gráfico, corresponderá àl que ha sido asignado en el texto, siempre acompañado de un título breve que apoye la comprensión de los resultados. ${ }^{\text {"I }}$ 


\subsection{Pertinencia del tema}

De acuerdo con lo expresado por Cunninghan y Day, el primer aspecto a considerar en todo articulo cientifico, que aspira a ser publicado, es la pertinencia del conocimiento que se desea divulgar, y en este sentido es importante evaluar el tema, su originalidad y su impacto tanto a nivel de la práctica clínica, como sobre el conocimiento existente. ${ }^{2.3}$

\subsection{Autoria}

Solamente pueden considerarse autores a aquellas personas que han hecho una contribución significativa en la elaboración del articulo. ${ }^{3}$ De acuerdo con lo estipulado en las Normas de Vancouver, son tres los requisitos que deberán cumplirse en forma simultánea y son:

- La concepción y el diseño del estudio o el análisis y la interpretación de los datos.

- La redacción del artículo o la revisión crítica de una parte sustancial de su contenido intelectual

- La aprobación final de la versión que será publicada."

\section{EL ARTICULO ORIGINAI.}

\subsection{Estructura}

La forma usual incluye la introducción, materiales y métodos, los resultados y la discusión y se conoce como el sistema IMRYD; ${ }^{24}$ es importante destacar que dentro de los aspectos preliminares se incluye el título y el resumen. pues también son elementos importantes del artículo original, al igual que las conclusiones y la bibliografia que se presentan al final del mismo.

\subsubsection{Título}

Permite al lector establecer la naturaleza del artículo y decidir si desea leerlo. Este elemento es particularmente importante, pues gracias a los nuevos sistemas electrónicos podría ser leído por miles de personas. Por lo tanto, deben elegirse con cuidado las palabras que lo integran y cuidar la forma de asociarlas. Un buen título es aquel que con el menor numero de palabras describe en forma adecuada el contenido de un artículo; ${ }^{2}$ no es extraño que el título sufra modificaciones durante el proceso de redacción del manuscrito.

Entre los errores frecuentes está ser demasiado extenso (exceder de 15 palabras). demasiado breve (no orienta ni dice nada), exceso de preposiciones y de artículos, y el uso innecesario de título y subtitulo. ${ }^{12}$

\subsubsection{Resumen}

Es una versión condensada del artículo (no debe superar las 250 palabras) y permite al lector identificar rápida y exactamente el contenido del documento, determinar su pertinencia para sus intereses y decidir si debe leer todo el artículo. ${ }^{2}$ Puede presentarse en forma estructurada 0 no estructurada. En la forma estructurada se resaltan los títulos esenciales del proceso investigativo como son objetivo, materiales y métodos, resultados y conclusión. Se expresan de la manera más sucinta los aspectos esenciales de cada una de ellas, es quizás la mejor forma de presentar un resumen para un articulo original. En la forma no estructurada se plantea como un párrafo continuo que explica en forma general la información relacionada con el trabajo. ${ }^{3.11}$

El resumen es un aparte del articulo que se escribe al final y cuando todo el proceso de elaboración de este ha terminado: adicionalmente se debe incluir el resumen en inglés o abstract y su redacción debe ser revisada por una persona experta. Es recomendable que al escribir en inglés, se utilice la forma activa, no se traduzca literalmente del español. ${ }^{5}$

Las palabras claves se ubican después del resumen tanto en español como en inglés: son palabras que permiten identificar el artículo en cualquier sistema de bủsqueda electrónica. Usualmente se usan de tres a cinco palabras o frases cortas, deberían emplearse los términos definidos en la lista del Medical Subjects Heading (MESH) del Index Medicus."

\subsubsection{Introducción}

Es la primera sección del texto propiamente dicho, su finalidad es la de suministrar suficientes antecedentes para que el lector pueda comprender y evaluar los resultados del estudio, sin necesidad de consultar publicaciones anteriores sobre el tema. Debe presentar el fundamento racional del estudio y por encima de todo, hay que manifestar breve y claramente cuál es el propósito del estudio. ${ }^{2.4}$

El objetivo de la investigación debe estar en el último párrafo de la introducción para indicar que la investigación ha sido desarrollada y orientada a responder al problema que se habia formulado al inicio. ${ }^{13}$

\subsubsection{Materiales y métodos}

Es la parte más importante del artículo científico porque proporciona la información que dará la validez al 
estudio." Se debe brindar suficiente evidencia sobre lo que se hizo y de esta forma el estudio podria repetirse. ${ }^{23.13}$ Es dificil lograr un balance adecuado entre la brevedad y la claridad, de modo que el lecror pueda responder las siguientes preguntas:

¿Qué tipo de estudio se realizó? Experimental u observacional. Si fue observacional, fue analítico o descriptivo. El tipo de estudio debe ser coherente con el problema y los objetivos y al valorar este punto, debe observarse que el estudio seleccionado permita alcanzar el propósito que se planteo en la investigación.

¿Quiénes integran el universo y cuántos integran la muestra? Es importante dar claridad a quienes se incluyeron. como fueron reclutados y donde se llevó a cabo el estudio para permitir valorar si los resultados del estudio pueden ser generalizados y si los sujetos involucrados representan a la población de interés. Además, se orienta al lector sobre la aplicabilidad clínica de los resultados. ${ }^{23}$

El número de sujetos que integran la muestra determina la probabilidad de detectar diferencias estadísticamente significativas entre los grupos que se comparan y estar razonablemente seguros, de que las que no se observaron es porque no están; esto es lo que se conoce como el poder del estudio y es un aspecto fundamental que debe evidenciarse en el texto del artículo. Para el cálculo del tamaño de muestra se debe especificar la fórmula, tabla o software que permitió determinarla. Finalmente, los mecanismos probabilísticos o no probabilísticos empleados para seleccionar los sujetos que integraron la muestra. ${ }^{4.78}$

¿Cuáles fueron los criterios de inclusión y exclusión que se consideraron en las personas involuctadas en el estudio? Deben brindar claridad de los sujetos investigados y de las posibles limitaciones que el estudio puede tener frente a las posibles generalizaciones que se pueden plantear: ${ }^{31, \pi}$

¿Qué variables se recolectaron? Se deben expresar en forma clara pero muy resumida e indicarse las bases cientificas que soportan su elección; si se emplearon indices validados. la operacionalización de las variables y las opciones de respuesta, los valores o rangos de valores que pueden ser alcanzados en cada una de las variables involucradas. ${ }^{3 \text { s }}$

¿Cuáles fueron los procedimientos básicos y que tipo de insumos se emplearon? Aqui debe hacerse evidente como se efectúo la prueba piloto y cómo se establecieron los mecanismos para garantizar la validez y la reprodu- cibilidad de los resultados; es necesario, además, exponer las intervenciones, seguimientos, enmascaramiento, asignación de grupos (experimental, control), mecanismos para determinar la selección de los grupos, equipos empleados, procesos de estandarización para que, de esta forma, se pueda evidenciar el control del sesgo y las variables que pudiesen confundir los resultados. ${ }^{1,8}$

¿Qué pruebas estadisticas se aplicaron? ¿Son las más adecuadas? Se hace necesario dar claridad en el análisis univariado del cálculo de las medidas de resumen de acuerdo con la naturaleza de la variable. Para el análisis bivariado. las pruebas estadisticas que permitan establecer asociaciones o diferencias entre los grupos que se comparan y su elección de acuerdo con la naturaleza de la variable, con la distribución de los datos y con la independencia de las mediciones. Si se aplican modelos de análisis multivariado, los supuestos que soportan los mismos. la importancia de este aparte radica en que la aplicación de métodos estadísticos inapropiados puede generar resultados erróneos, por lo que debe ser muy claro.'?

¿Qué consideraciones éticas se tuvieron en cuenta? Se resalta cómo la investigación se acoge a las recomendaciones éticas establecidas en la resolución 008430 y que incluyen la aprobación de un Comite de Ética Institucional y cómo se preservan los principios de beneficencia, autonomía, privacidad y confidencialidad. ${ }^{14}$

\subsubsection{Resultados}

Para algunos, esta sección se considera el corazón del articulo. Es la razón por la cual se busca la publicación del articulo. ${ }^{13}$ Deben presentarse los datos sobre los que el estudio se fundamentó; es necesario que se encuentren en un orden lógico, con los datos básicos y los análisis más elementales antes de las comparaciones y los análisis más complejos, de acuerdo con lo planteado en el plan de análisis estadistico. ${ }^{t 16}$

Su formato debe ser claro y conciso, no se deben incluir los antecedentes. Los datos se exponen en textos, tablas, gráficos y/0 ilustraciones. Se espera que los resultados presentados en forma gráfica (tablas o gráficos) se resuman en forma muy breve en el texto. con el propósito de apoyar al lector en su comprensión y de esta forma resaltar la información más relevante. En la tabla 1 se pueden observar las ventajas y desventajas de las diferentes formas de presentación de los resultados. 


\begin{tabular}{|c|c|c|c|c|}
\hline & Texto & Tabla & Gráfico & Ilustración \\
\hline Contenido & $+t+$ & $+++t$ & $+t$ & + \\
\hline Precisión & $+t+$ & +++ & ++ & + \\
\hline Impacto & + & ++ & ++++ & $+t+$ \\
\hline Intereses & + & ++ & +++ & ++++ \\
\hline
\end{tabular}

Tabla 1. Caracteristicas de las formas de presentación de datos en las publicaciones cientificas.

En las tablas no se debe presentar gran cantidad de información que dificulte al lector su comprensión; la presentación de la información en gráficas busca ilustrar las tendencias observadas y no la de proveer datos específicos. de ahí la importancia de que sean sencillas y fáciles de comprender. Cada tabla y gráfico debe tener un titulo corto y claro y en los pies de tabla indicar aspectos relacionados con las pruebas estadisticas aplicadas, el nivel de significancia y la fuente de los datos. ${ }^{2,7}$ Las ilustraciones pueden ocasionar gran impacto pero, generalmente, no proveen una clara información.?

\subsubsection{Discusión}

Puede ser la sección más dificil de construir. Debe incluir un resumen crítico de los principales resultados del estudio; su objetivo es poner los resultados dentro de una perspectiva. ${ }^{3,13}$ es decir, expresar las posibilidades de generalizar los resultados y sus implicaciones clinicas. Por lo tanto, es importante vincular la información obtenida con trabajos previos relacionados con el tema y a la luz de los de los factores que podrian sesgar los resultados.

En los temas donde hay una buena base de conocimiento, el autor debe hacer énfasis en los estudios que soportan sus resultados y aquellos que no. También, se permite especular sobre el impacto de los hallazgos. ${ }^{15}$

Los errores más frecuentes son: repetir los resultados, no confrontar los resultados, reformular los puntos ya tratados, polemizar en forma trivial, hacer comparaciones teóricas débiles, especular sin identificarlo como tal y sin relacionarlo en forma estrecha y lógica con informa ción empírica o teórica. ${ }^{17}$

Además, es importante reconocer las limitaciones potenciales del estudio. pues ningún artículo es perfecto, por lo que dichas limitaciones no deben ser ignoradas. ${ }^{3}$

\subsubsection{Conclusiones}

El articulo debe finalizar con cuatro o cinco conclusiones relevantes del trabajo con el propósito de generar mayor impacto en los lectores. ${ }^{3}$ En esta sección, es posible hacer las recomendaciones que se consideren convenientes de acuerdo con la investigación realizada. ${ }^{15}$

\subsubsection{Bibliografia}

La validez de las referencias es responsabilidad del autor o autores y debe verificarse cuidadosamente. Los pares evaluadores, usualmente, seleccionan una o dos referencias al azar y las corroboran; si hay inconsistencias en las referencias podría dar la impresión que se está frente un artículo de mala calidad. ${ }^{3}$

Cada revista ha seleccionado su sistema de referencia. Existen fundamentalmente dos tipos de citación bibliográfica:

- Sistema recomendado por el Comité Internacional de Editores de Revistas Biomédicas: se caracteriza por utilizar superindices secuénciales en el texto y las referencias se presentan en el orden de citación." Los superin-dices son escritos después del punto que termina una idea. ${ }^{5}$

- Sistema de Harvard: usa el nombre del primer autor y los datos del artículo, la bibliografia se organiza en orden alfabético. ${ }^{3}$

Se debe evitar tomar referencias de otros articulos sin tener el articulo original, pues pueden existir interpretaciones invalidas de un autor en trabajos impresos previos. ${ }^{35}$

Los errores más frecuentes se presentan cuando no se citan las referencias o se citan equivocadamente, éstas son obsoletas y muestran desactualización del autor, se seleccionan sin cuidado, son muy numerosas lo que muestra pseudo-erudicción del autor, se incluyen referencias no consultadas personalmente por el autor y los documentos que respaldan las referencias no son accesibles al lector. ${ }^{17}$

\section{EL ARTÍCULO DE REVISIÓN}

Suele ser extenso, es común que tenga entre 10 y 50 paginas impresas. La revisión bibliografia es, desde luego, el producto principal. Un buen artículo de revisión ofrece una evaluacion crítica de los trabajos publicados y llega a conclusiones importantes basado en estos trabajos. De los mejores artículos de revision surgen nuevas sintesis, ideas, teorías e incluso nuevos paradigmas. ${ }^{24}$

52

Ustasalud Odontologia 2005; 4: 48 - 55

Concha SC. y col. 


\subsection{Estructura}

\subsubsection{Titulo}

Guarda los mismos esquemas establecidos para el artículo original y en él se puede hacer evidente o no que se trata de una revisión.

\subsubsection{Resumen}

Preserva muchas de las caracteristicas establecidas en el artículo original; sin embargo, es importante destacar que el resumen, en los artículos de revisión, expresa brevemente el problema, el objetivo, el método utilizado para estudiar la información más relevante y las conclusiones. Usualmente, se usa la modalidad no estructurada que debe tener un máximo de 150 palabras. 5

\subsubsection{Introducción}

En los artículos de revisión, esta sección es especialmente amplia: en ésta se deben establecer claramente los objetivos, las razones para el estudio para permitir al lector valorar, si se investigó en un tópico importante. ${ }^{2}$

\subsubsection{Métodos}

Este punto en particular puede ser opcional y su inclusión depende fundamentalmente de las exigencias establecidas por cada publicación. Cuando este aparte se incluye en el articulo de revision, se describe cómo se ha hecho la revisión bibliográfica, las fuentes usadas y los métodos de selección de los articulos. ${ }^{5}$

\subsubsection{Cuerpo del articulo de revisión}

A diferencia de lo que se observa en los artículos originales, no hay una organización establecida para los de revision; por consiguiente, se debe elaborar una guia propia que puede ser por títulos y subtitulos para mayor compresión del documento.

El lenguaje con que se desarrolla tanto el cuerpo, como cada uno de las secciones que constituyen el artículo de revisión deberá ser sencillo pues el público al cual va dirigido es muy variado. ${ }^{2}$

\subsubsection{Anexos}

En algunos casos es útil manejar la información a través de tablas, dibujos o esquemas. Se debe tener en cuenta que los anexos han de ser originales y que en el caso de ser obtenidos de otra fuente, debe existir el reconocimiento adecuado.

\subsubsection{Conclusiones}

Como se abarca un tema amplio, las conclusiones se constituyen en un componente fundamental y es especialmente importante cuando se trata un tema de alta complejidad técnica, novedoso o confuso. Deben plantearse en forma ordenada, clara y que resuman los aspectos básicos que se presentaron en el documento respondiendo al problema y a las preguntas que dieron origen a la revisión y haciendo evidente que se cumplió a cabalidad con el objetivo propuesto. ${ }^{2}$

\subsubsection{Bibliografia}

Se deben considerar las normas establecidas por cada revista. El artículo de revisión por su misma naturaleza de recopilación, exige una excelente bibliografia. Day afirma que un articulo de revisión debería estar sustentado con, por lo menos, 50 referentes bibliográficos. ${ }^{2}$

\section{EI. REPORTE DE CASO}

Se enmarca dentro del diseño descriptivo de la investigación clinica. ${ }^{6}$ Generalmente, se publican si el caso contribuye a un cambio en el curso de la ciencia; si muestra un principio nuevo o apoya o refuta una teoria; si demuestra una respuesta adversa a una terapia medicamentosa, y si se observa una combinación inusual de condiciones no estudiadas. $^{18}$

\subsection{Estructura}

Su estructura es similar a los artículos ya descritos. Un reporte de caso tiene seis secciones: titulo, resumen, introducción, descripción del caso, discusión y bibliografia. ${ }^{19}$ Solo se realizará énfasis en los aspectos adicionales a los ya tratados.

\subsubsection{Resumen}

Debe ser corto, simple, claro y explicativo por sí mismo. Se realiza en un solo párrafo que no exceda las 150 palabras. La simplicidad demanda el uso de frases cortas; debe proporcionar una breve explicación de la introducción, del reporte de caso, de la conclusión y del objetivo que se quiere lograr al presentarlo. No se deben colocar apartes de la discusión en el resumen, tampoco lleva citas bibliográficas. ${ }^{19}$

\section{1 .2 Introducción}

Se realiza una breve revisión del tema y se muestra el por qué era necesaria la publicación del reporte. Las afirmaciones deben estar sustentadas en referencias bibliográ- 
ficas. Puede ser de uno, dos o tres párrafos. ${ }^{18}$ Los párrafos no deben tener más de diez frases (excepto en el resumen) y no menos de tres; cada párrafo debe tener una secuencia lógica de modo que la idea central sea desarrollada a través de éste. La última frase de cada párrafo debe tener una conexión con la primera del siguiente..$^{19}$

\subsubsection{Descripción del caso}

Se cuenta la historia del caso. En la primera frase se debe relatar el género del paciente, su edad y su motivo de consulta o queja principal. A partir de ésta, se inicia la redacción de todo el caso. En la mayoria de los casos es necesario presentar la descripción del paciente, la historia de su condición actual, el examen fisico, los exámenes de laboratorio que son relevantes, el diagnóstico, el tratamiento, las expectativas de su condicion y su estado actual. ${ }^{18.19}$

\subsubsection{Discusión}

Como en los demás tipos de artículos, en éste tampoco se repite la introducción. Es muy importante el orden de los párrafos y se secuencia. Alfonso sugiere qque la discusión se presente de la siguiente manera: ${ }^{1 y}$

- El párrafo inicial debe proporcionnr una discusión breve de los diagnósticos diferenciales basados en los hallazgos observados.

- El segundo párrafo explica las bases del diagnóstico y la patología o entidad como tal.

- El tercer párrafo discute la información publicada que apoya el manejo realizado.

- El cuarto párrafo crea el debate con antecedentes contradictorios.

- El quinto párrafó concluye.

\section{ASPECTOS FINALES RELACIONADOS CON EL AR- TíCULO}

En esta fase es útil solicitar a uno o varios colegas la lectura del articulo con el fin de verificar la calidad y comprensión del mismo; es importante dejarlo a un lado durante un corto lapso de tiempo y volver a leerlo más adelante. ${ }^{3 . t}$

Se debe verificar en los textos, las tablas y los grálicos cualquier tipo de inconsistencia, los modismos, las muletillas y cualquier aspecto que pueda ser ambiguo. Se corrobora nuevamente que se cumplen con las instruccio- nes para los autores de la revista en la que se quiere publicar. Finalmente, se envía a la revista; generalmente, se debe esperar entre tres o cuatro semanas para que la persona responsable de acuso de recibo del manuscrito.?

En el caso de que el artículo sea devuelto para correcciones, éstas deben efectuarse con la mayor rapidez posible y cada punto solicitado para corrección debe ser analizado y discutido con cada uno de los coautores. Posteriormente, se debe escribir una carta detallada de su respuesta frente a las modificaciones solicitadas. ${ }^{3.3}$

Cuando el artículo ya ha sido aceptado, usualmente la revista envía al autor una reproducción previa del artículo (prueba o galerada) con el fin de que se verifique cualquier error. Es responsabilidad del autor corroborar que la versión final de la publicación no tenga ningún tipo de inconsistencia, especialmente a nivel de tablas y de referencias bibliográficas. ${ }^{3}$ El diagrama que sugiere Rosenfeldt y colaboradores muestra el flujograma que usualmente se desarrolla cuando se escribe un manuscrito para una publicación científica (Figura 1).

\section{CONCLUSIONES}

- La divulgación del conocimiento cientifico es una parte esencial dentro del proceso investigativo. Por lo tanto, es necesario disponer de las normas técnicas que permitan hacer una presentación o escribir un articulo con todas las especificaciones técnicas necesarias para lograr este propósito.

- Existen diferentes formas de publicación, dentro de éstas se pueden destacar los artículos originales, los articulos de revisión y los reportes de casos. Cada uno de estos tiene una función especifica dentro del proceso de divulgación del conocimiento y asi mismo una estructura particular que debe ser adoptada si se espera que las revistas cientificas acepten el manuscrito.

- El objetivo central de un artículo original es proveer nuevo información que permita un mayor desarollo en el área analizada. El artículo de revisión por su parte, retoma el conocimiento ya existente y se estructura de tal forma que permita una mayor comprensión o una recontextualizacion sobre el tema tratado. El reporte de caso se considera como la semilla para formular nuevas preguntas. 


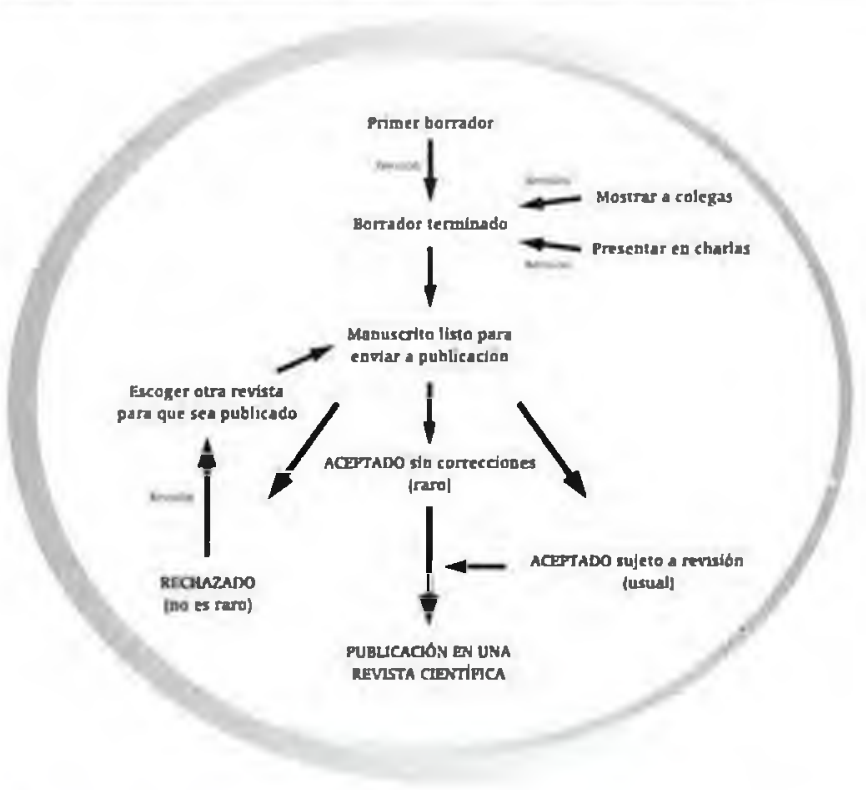

Figura 1. Flujograma para el desarrollo y publicación de un manuscrito.'

- Independientemente del tipo de manuscrito que se produzca, éste debe acogerse a las normas establecidas por cada revista y asi mismo brindar a los colegas la oportunidad de comprender, replicar y aplicar el nuevo conocimiento en el contexto en el que se desenvuelve su ejercicio profesional.

\section{BIBLIOGRAFIA}

1. Asociación Colombiana de Facultades de Odontologia. División de Investigación. Hacio el desarrollo de una cultura investigativa con responsabilidad social. Politica y plan estratégico 2003-2007. Medellin. COIY @NET. Comunicoción integral p.11.14

2. Day AR. Cómo escribir y publicar trabajos cientificos, 3era. Edición Pan American Health Organization, 2003.

3. Cunningham S]. How to...write a paper. J Orthod 2004; $31: 47$ - 51. 4. Navarro ]. Taller sobre redacción de articulos cientificos. Organización Panaınericana de la Salud. Lima, 25 y 26 de mayo de 2004.

5. Delgado JE. Hil articulo de revisiön: guio para su escritura. Univ Odontol 2002: 22: 77 - 79 .

6. Delgado JE. Los estudios de caso: conceptos generales y guía para la escritura de reportes de caso y series de casos. Univ Odontol 2002; 22: $91-94$.

7. Rosenfeldı FI. Dowling JT, Salvatore P, Fullerton M]. How to write a paper for publication. Heart, l.ung and Circulation 2000:9:82 - 87 .

8. Kallet RH. How to write the methods section of a research paper. Respir Care 2004; 49: $1229-1232$.
9. Albert T. Cómo escribir artículos científicos fácilmente. Gac Sanit 2002: 16: 354 - 357.

10. Jiméne: ME. Preocupaciones actuales sobre la confección de titulos adecuados para articulos científicos. Medisan 2004: 8: 42 - 45.

11. International Committee of Medical Journal Editors. Uniform requirements for manuscripts submitted to biomedical journals. JAMA 1993: 269: $2282 \cdot 2286$.

12. Jara E. La selección del título en el artículo cientifico. Rev Cubana Med Gen Integr 1999: 15: 342 - 345.

13. Harrison JE. Fvidence based orthodontics - How do I assess the evidence? J Orthod 2000: 27: $189-197$.

14. Republica de Colombia. Ministerio de Salud. Resolución $008430 \mathrm{de}$ 1993.

15. McCabe BF: Editorial: How to write a scientitic paper. 1989. Ann Otol Rhinol Laryngol 2004: 113: 531 - 532.

16. Wells WA. Me write pretty one day: how to write a good scientific paper. J Cell Biol 2004; 165: 757 - 758.

17. Bobenrieth MA, Ribbi-jaffe A. Partes del articulo cientifico. Was hington. DC: Organizaciỏn Panamericana de Salud, Organización Mundial de Salud. 1990: 3 - 4.

18. McCarthy LH, Reilly K. How to write a case repor. Fam Med 2000; 32: $190-195$.

19. Alfonso I. Case reports: Why, what and how. Int Pediatr 2000; 15: $44 \cdot 50$
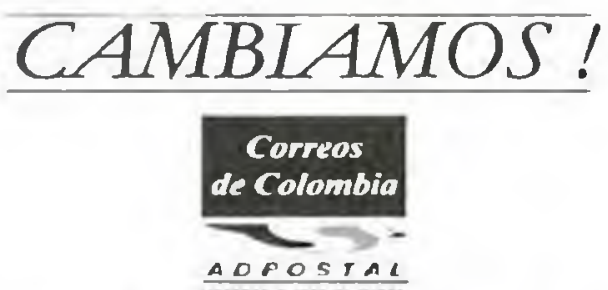

Pensando en ofrecerle el mejor servicio

Nuestras Lineas de Atención al Cliente

$4298487-2633484-2956898$

$018000111210 / 111313$

Fax: 4163028

Sabgerencia de Meradioo

3340304

Divivibo de Merandoo Regional DC

4297320

WWw.adpostal.gov.co

\section{西}

